\title{
Analyzing problem solving using math in physics: Epistemological framing via warrants
}

\author{
Thomas J. Bing \\ Department of Physics, Emory University, Atlanta, Georgia 30322, USA \\ Edward F. Redish \\ Department of Physics, University of Maryland, College Park, Maryland 20742, USA
}

(Received 25 July 2009; published 14 December 2009)

\begin{abstract}
Developing expertise in physics entails learning to use mathematics effectively and efficiently as applied to the context of physical situations. Doing so involves coordinating a variety of concepts and skills including mathematical processing, computation, blending ancillary information with the math, and reading out physical implications from the math and vice versa. From videotaped observations of intermediate level students solving problems in groups, we note that students often "get stuck" using a limited group of skills or reasoning and fail to notice that a different set of tools (which they possess and know how to use effectively) could quickly and easily solve their problem. We refer to a student's perception or judgment of the kind of knowledge that is appropriate to bring to bear in a particular situation as epistemological framing. Although epistemological framing is often unstated (and even unconscious), in group problem-solving situations students sometimes get into disagreements about how to progress. During these disagreements, they bring forth reasons or warrants in support of their point of view. For the context of mathematics use in physics problem solving, we present a system for classifying physics students' warrants and analyze a case study. This warrant analysis provides a general widely applicable technique for identifying students' epistemological framings.
\end{abstract}

DOI: 10.1103/PhysRevSTPER.5.020108

PACS number(s): 01.40.Fk, 01.30.1b

\section{INTRODUCTION}

\section{A. Motivation}

Mathematics is the backbone of physics. It provides a language for the concise expression and application of physical laws and relations. A student's development as a physicist entails, in no small part, becoming increasingly comfortable with mathematics. As physics teachers, we share a responsibility to help our students develop fluency with the mathematics of physics. But what does it mean, "to become comfortable with mathematics in physics," how would we recognize it happening in a student, and how, as instructors, can we facilitate this process? In this paper, we describe how we develop a clearer understanding of this issue by using videotaped ethnographic observations of groups of upperlevel physics students solving physics problems.

What we learn is that although mathematics is an essential component of university level science, math in science is considerably more complex than the straightforward application of rules and calculation taught in math classes. Using math in science critically involves the blending of ancillary information with the math in a way that both changes the way that equations are interpreted and provides metacognitive support for recovery from errors. There are many different ways to interpret mathematics in physics - and students often need to blend physical, mathematical, and computational reasons for constructing and believing a result.

In order to analyze student behavior along this dimension, we have used the analytical tool of epistemological framing. This refers to the student's perception or judgment (unconscious or conscious) as to what class of tools and skills is appropriate to bring to bear in a particular context or situation. Although these framings are often tacit, in one particular situation they become much more explicit: when students argue with each other about what to do next. When there is a disagreement, the discourse tends to include warrantsreasons for drawing a conclusion. We demonstrate that these warrants fall into clusters, casting a light on the way the student has framed the situation epistemically. This leads us to create system of classifying warrants that should provide a useful lens on the development of problem-solving skills.

Our observations of students indicate that epistemological frames can be "sticky;" that is, students can get trapped in them for relatively long periods of time (many minutes). These observations suggest that an epistemological framing analysis can be potentially useful for instruction. First, it can suggest activities that might facilitate the development of expertise; second, it can permit an instructor to recognize the development of sophisticated problem-solving behavior even when the student makes mathematical errors. ${ }^{1}$

\section{B. Example}

Many physics students struggle as they try to develop mathematical fluency. Part of their struggle is undoubtedly due to the sheer conceptual complexity of the mathematics commonly encountered in physics classes. As a physics major progresses through the undergraduate curriculum, she will encounter techniques requiring series expansions, threedimensional vector calculus, linear algebra, complex numbers, differential equations, probability theory, and more. A robust understanding of any of these topics involves a complicated coordination of a large amount of information. University math departments commonly devote at least a semester-long class to each of the topics on that list.

This paper, however, focuses on a different aspect of the mathematical complexity our physics students encounter: epistemic complexity. "Epistemic" is an adjective referring 
to what one knows about the nature of (in this case, mathematical) knowledge. Simply put, the same piece of mathematics commonly fills many different roles in a physics class.

To illustrate epistemic complexity, consider the expression $x_{f}=x_{i}+\langle v\rangle \Delta t$, relating an initial position $x_{i}$, a final position $x_{f}$, an average velocity $\langle v\rangle$, and a time interval $\Delta t$. First, this expression encodes a calculational scheme. If the object starts at $x_{i}=3 \mathrm{~m}$ and then maintains an average velocity of $\langle v\rangle=4 \mathrm{~m} / \mathrm{s}$ for $\Delta t=2 \mathrm{~s}$, then the expression $x_{f}=x_{i}+\langle v\rangle \Delta t$ tells you how to combine those given values to produce a numerical result for $x_{f}: x_{f}=(3 \mathrm{~m})+(4 \mathrm{~m} / \mathrm{s})(2 \mathrm{~s})=11 \mathrm{~m}$.

Second, $x_{f}=x_{i}+\langle v\rangle \Delta t$ encodes a physical relation among measurements. An average velocity tells how far an object travels per given length of time. Multiplying by the time of the journey gives $\langle v\rangle \Delta t$, unwinding the definition of $\langle v\rangle$ and representing how far you move in a given time interval. Tacking that on to where your object started from, $x_{i}$, must yield the position at the end of the journey, $x_{f}$.

Third, mathematics provides us with a concise system for recalling encoded rules and previously derived results. No one starts all physics problems from first principles every time. One can imagine a physicist simply quoting that $x_{f}=x_{i}+\langle v\rangle \Delta t$ and simply thinking to himself "that's what the final position equation is."

Fourth, our sample expression $x_{f}=x_{i}+\langle v\rangle \Delta t$ can be seen filling yet another role, highlighting another epistemic feature of mathematics in physics. It fits in with a large interrelated web of mathematical ideas. For example, it can be derived from the definition of average velocity by simple algebraic manipulation. It also has the conceptual structure of a base-plus-change symbolic form, ${ }^{2}$ just like the equation $v_{f}=v_{i}+\langle a\rangle \Delta t$, and it coordinates with the interpretation of the determination of distance traveled from a velocity graph by the calculation of the area under the curve. Stepping even farther back, $x_{f}=x_{i}+\langle v\rangle \Delta t$ can be seen as a solution of a differential equation of the form $d^{2} x / d t^{2}=k$.

Mathematics thus fills many different epistemic roles for a physicist. It reflects physical relations, provides a calculation framework, forms a web of interconnected ideas, and provides a packaging system for encoding rules and previous results. Even such a simple expression as $x_{f}=x_{i}+\langle v\rangle \Delta t$ displays this epistemic complexity.

This paper discusses a detailed case study of a group of upper-level undergraduate physics students at work on a homework assignment in their physics class. These students are grappling with the epistemic complexity of the mathematics, often struggling as they juggle various interpretations of the math at hand. Our goal is to model their thinking.

In Sec. II we discuss the cognitive framework we are working in including giving an explanation of the concept of framing. We also give a brief review of the relevant elements of argumentation theory that inform our framing analysis. In Sec. III we discuss our methodology. In Sec. IV we identify the four epistemological framings we find in our data, and in Sec. V we present our detailed case study. We present our conclusions and the implications for instruction and future research in Sec. VI.

\section{THEORETICAL BACKGROUND}

We begin by situating ourselves within a particular theoretical way of thinking about student thinking: the resource framework (RF). We give a brief summary of the assumptions of this approach. We then provide a simple example ("Sarah shifts her reasoning") to illustrate how epistemological framing fits within the RF. We then discuss framing in general and epistemological framing in particular.

\section{A. Resource framework: A brief overview}

There are many theoretical lenses that are available for building models of student behavior. The one we use is the RF that has evolved out of the "knowledge in pieces" approach developed by diSessa and his collaborators. ${ }^{3,4}$ This framework is documented in detail in a number of published papers. ${ }^{5-8}$ We present a brief overview here but encourage readers interested in more detail to access the original papers.

The RF is a structure for creating phenomenological models of high-level thinking. It is based on a combination of core results selected from educational research phenomenology, cognitive or neuroscience, and behavioral science. It is a framework rather than a theory in that it provides ontologies-classes of structural elements and the way they behave-and it permits a range of possible structures and interactions built from these elements. As such, it provides a framework that permits the creation of descriptive and phenomenological models that bridge many existing models such as the alternative conception theory and the knowledge in pieces approach or cognitive modeling with the sociocultural approach. The RF does not (as yet) create mathematical models in which predictions arise from calculations.

The RF is an associative network model with control structure and dynamic binding.

\section{Associative network}

The basic ontology of the RF is that of a network, built from the well-established metaphor of neurons in the brain. The activation of the neurons corresponding to one resource or cluster of resources leads to activation of other clusters. Learning is pictured as the establishment of strong connections, so that activation of one resource or cluster of resources leads to the activation of other resources. Associations can be excitatory (encouraging activation) or inhibitory (discouraging activation).

\section{Control structure}

The network of resources in the brain is not simply associative. The brain has structures (hippocampus, cingulate gyrus, etc.) that appear to have specific purposes, just as in other parts of the body (heart, lungs, liver, etc.). In the prefrontal cortex perceptual information is mixed with longterm memory to prime appropriate actions. The evaluation of a perceived situation affecting action is a well documented component of behavior in mammals. (See, for example, Fig. 1 in Ref. 9 and the extensive references there.) Control structures rely heavily not only on activating association but also on inhibition. 


\section{Binding}

Clusters of resources that activate together frequently become strongly tied so that they always activate together. ${ }^{10}$ This makes possible the creation of networks of higher level structures-concepts, p-prims, or schemas-that the user perceives as unitary. Binding can occur at many levels from being extremely tight (e.g., it is hard to see the word "cat" and not to imagine its referent) to being rather loose (e.g., one can perceive an orchestral performance as unitary or listen for individual instruments or motifs). Both basic associations (concepts) and control structures (framings) can be tightly or loosely bound.

\section{Dynamic}

A critical element of the entire model is that it is extremely dynamic. Associations are activated and inhibited depending on context.

In this paper we are building a component of the control structure (i.e., how the mind allocates its power) appropriate for modeling students' use of mathematics in physics: epistemological framing.

\section{B. A framing story: Sarah shifts her reasoning}

An analysis in terms of epistemological framing focuses on the moment-to-moment shifts observed in students' reasoning. Their interpretation of the task and knowledge at hand can change, as in the following example. ${ }^{11}$

"Sarah" is an upper-division undergraduate physics major who sat for an interview aimed at her understanding of electrical conductors and insulators. Sarah has just explained how insulators are so dense that current cannot flow through them. Wanting to explore this further, the interviewer brings up the case of Styrofoam. When the interviewer asks her whether Styrofoam is an insulator, Sarah responds that it is. Her response to the interviewer's question "Why?" is that she "memorized it." The conversation continues, and when the next opportunity arises for Sarah to justify a claim she makes a blanket statement citing "organic chemistry." So far in the interview, she is relying on authority in her explanations, quoting rules and facts.

After the interviewer prods her to give "any explanation you find," Sarah's reasoning undergoes a shift. She gives a more detailed, more conceptual account of conductance. Sarah puts together a little story about electrons getting torn away from their parent atoms and then being free to move. She explains how a battery could perhaps cause this electron tearing and how a higher temperature wire might also have more energy available to tear electrons off the atoms.

The shift we care about in Sarah's reasoning concerns the types of explanations she gives. She began by quoting facts. Implicit was Sarah's epistemic interpretation of her situation and the interviewer's intentions. What is the nature of the knowledge in play here? "Oh, OK, this interviewer wants factual information about conductors and insulators. I'll give him some facts I remember."

The interviewer's apparent dissatisfaction with her quoted facts and subsequent "any explanation you find" prompt caused Sarah to reinterpret the activity. She came to see the interviewer's questions as prompts to tell a story about conduction. Sarah is less sure of her story about tearing off electrons than she was about her quoted facts, but she sees this uncertainty as permissible now. Epistemically, "now we're constructing stories, not quoting facts."

Briefly, Sarah has a different epistemological framing of her activity in the two parts of this episode. The different epistemological framings, different implicit answers to "What kind of knowledge is in play here?" led Sarah to bring different subsets of her knowledge store to bear on the interviewer's questions.

We now turn to a more detailed account of this epistemological framing process, beginning with an overview of epistemic resources.

\section{Epistemological resources activate and deactivate in Sarah's reasoning}

In our brief example Sarah treats knowledge as two different types of things. She begins by viewing knowledge as fact based and authority driven, later shifting to seeing knowledge as a personally constructed thing. We describe this shift by saying that different epistemological resources have been cued in Sarah's mind.

An epistemological resource is a cognitive modeling element. It represents a tightly bundled packet of information that, when activated by the mind, leads the individual to interpret the knowledge at hand in a certain light. But an epistemological resource is a control structure, not a concept; epistemological resources affect how students perceive the nature of the situation under current consideration and they control what conceptual resources are brought to bear. Are the students seeing scientific knowledge as fixed and absolute or as being relative to one's point of view? Or do they view scientific knowledge as something they can construct for themselves or as something that must be handed down from an authority figure? ${ }^{12,13}$

Epistemological resources, like other resources, are dynamic; they can activate and deactivate during the momentto-moment flow of an episode. Broad decontextualized questions such as "Do you see science knowledge as being handed down from authority?" at least by themselves are unlikely to elicit meaningful information on students' functional epistemologies. Such a question assumes that students have relatively stable context-independent beliefs about the nature of science. Much like the case with conceptual knowledge, ${ }^{3,6}$ authors have argued that students' epistemic stances are manifold and highly sensitive to context. ${ }^{14,15} \mathrm{Sa}-$ rah, for example, displayed a shift from "knowledge as authority driven" to "knowledge as constructed by oneself" in her brief electric conduction interview. This shift happened in response to an interviewer's prod. It was an in-themoment reaction to the natural flow of the conversation. One would certainly not expect that this isolated shift signals a large-scale change in Sarah's approach toward physics. It is unreasonable to think she never saw physics as being about telling conceptual stories before nor is it reasonable to think she will never quote authority again. There are many similar published examples of in-the-moment shifts in students' reasoning. $6,7,16,17$ 
As further evidence of the manifold nature of students' epistemologies, there also tends to be a disconnect between how students view the nature of formal science and how they proceed to interpret their own work in science class. ${ }^{18}$ Epistemological stances evolve, in a time averaged sense, in complex ways as students progress through their education. ${ }^{19}$

On more local time scales, many epistemological resources are available to students. These epistemological resources are often closely correlated with certain bits of the wide range of conceptual information available to students. Sarah's activation of knowledge as authority driven pointed her toward her store of organic chemistry facts-or perhaps vice versa.

With a wide range of conceptual and epistemological possibilities available, a model of students' thinking must also include a process by which the set of all possible epistemological and conceptual options is pared down to a manageable size for consideration by the individual. That process is called framing.

\section{Framing: What kind of activity is going on here?}

So far, we have been using the term framing in what amounts to a "common speech" mode. We have not defined it explicitly. But in the behavioral sciences, especially anthropology and sociolinguistics, framing represents a specific technical term. Framing is the, usually subconscious, choice the mind makes answering the following question: "what kind of activity is going on here?" It narrows down the set of all possible mental options to a manageable subset. An individual's framing of a situation tells them what is necessary to pay careful attention to in a situation and what can be considered irrelevant and ignored. This "selective attention" reduces processing load and is the benefit created by the mental structure that permits framing. ${ }^{20}$ Framing is a common pervasive cognitive process.

As a quick example, consider entering a hotel. Even if you have never been in that particular hotel before, you will immediately have a general idea how to proceed. You would expect there to be a front desk with a check-in clerk, lots of numbered rooms organized in a particular way, and perhaps a restaurant or two. You would plan on doing certain things in this building like sleeping and preparing for the next day's business. You would also have social expectations. You would not plan on shouting across the lobby, playing your television at full volume at 11 p.m., or throwing furniture off the balcony.

Framing should not be equated to activating a large stable instruction list. It is not as if you immediately run down a checklist upon entering a hotel. Where is the elevator? There it is. Where is the concierge desk? There it is. Where is the restaurant? There it is. Large data structures such as this hotel list are like set empty slots ready to be filled in with the particulars of a situation. Several early studies in artificial intelligence (from which modern framing studies partially evolved) were concerned with identifying (and then programming) such data structure "frames."21-23

This paper does not equate framing with the recall and activation of organized rigid data structures. Rather, we see framing as the cuing of fuzzy adaptable networks of cognitive resources. Not finding a restaurant in your hotel does not necessarily destroy your interpretation of your surroundings as a hotel. If the room numbers' organization is not the standard floor-by-floor numeric order, you would likely still be able to find your room eventually.

Framing has been studied in a wide array of academic disciplines including linguistics, sociology, art, psychology, and anthropology. ${ }^{24-27}$ All of these studies implicitly agree on the existence of what has been called "Felicity's condition." 28 Felicity's condition is the unspoken premise naturally adopted by an individual that incoming information, whether it be spoken, read, observed, etc., comes from a rational source, and it is thus up to the individual to attempt to contextualize and hence interpret that incoming information. Framing is the process by which the mind attempts this contextualization and interpretation.

Different individuals can certainly frame the same incoming information in different ways. ${ }^{26}$ A quick example is to note that what may be play to a golfer is work to the caddy. ${ }^{29}$ Miscommunications can arise when two individuals frame their interaction differently, each bringing a different subset of their available resources to bear on the situation. Framing should not be thought of as something that happens only once at the start of a new activity. People continually recheck their framing of a situation and may alter it accordingly, bringing new resources into conscious consideration while temporarily disregarding other ones. ${ }^{30}$ Sarah and her discussion of electrical insulators is one such example.

Framing can lead people to subconsciously disregard some strands of input information that are not seen as currently relevant. A latecomer taking his seat at a theater can be ignored, possibly not even noticed, by other audience members. ${ }^{25}$ The students in this paper's case study display an analogous selective attention. They can seem temporarily oblivious to a mathematical course of action that may be obvious to a classmate, instructor, or researcher. We interpret this selective attention as a result of their epistemological framing of their mathematics. They, in that moment, are interpreting the math at hand in a certain way, focusing on a particular aspect of the math knowledge in play.

\section{E. Argumentation theory helps us get evidence of framing}

Framing is often unconscious, even unnoticed by the person doing it. How, then, can a researcher gather evidence of how these upper-level physics students are framing their use of mathematics? How can we identify what they see as "the particular nature of the math knowledge in play"? A possible solution was suggested in our observations of students working on physics problems in groups. When students disagree on a procedure or result, they usually become relatively more explicit about why something should be believed or not. These comments help us identify what are referred to as warrants in argumentation theory. These warrants show us what epistemic assumptions they are making at the moment. We therefore suggest to look at the warrants they use in their math arguments. We hence turn to a brief overview of argumentation theory. 
There are several subfields that are sometimes colloquially lumped under the rubric "argumentation theory." 31 On one end of the continuum is what is best called formal logic. Logic chains like "if A then B, if B then C but not D, etc.," lend themselves readily to computational modeling, ${ }^{32}$ although even such apparently straightforward applications of classical logic rely on fuzzy mental processes that are very difficult to describe in detail analytically. ${ }^{33}$

A second branch of research, the one that is most often actually called argumentation theory, includes what is often called rhetoric. ${ }^{31}$ This field of research focuses most on presenting, as opposed to having, an argument. A central pillar of this field, and an important basis for this paper's analysis, is the work of Toulmin. He devised an often-cited system for parsing an argument into such parts as claims, data, and warrants. ${ }^{34}$ A person will make a statement, the claim, which requires proof. They will then offer one or more relevant facts, the data. The warrant is the bridge, sometimes unspoken, which explains how the given data relate to the claim at hand. For example, I might state that Thomas Jefferson is the greatest American founding father (claim) because he largely wrote the Declaration of Independence (data). The relevant warrant that would link these data to that claim would be that the Declaration of Independence is a cornerstone document of the United States, laying out the nascent country's case for autonomy.

Naturally occurring arguments are more nebulous than a formal logic structure allows. Nor should one expect a clean Toulmin structure to fit most ordinary arguments. ${ }^{35,36}$ Justifications that may be logically unsound can be cognitively sound-completely acceptable and compelling in informal real-time situations according to complicated probabilistic mental processes. ${ }^{37} \mathrm{~A}$ branch of research, often gathered under the label "discourse analysis," 31 concerns itself primarily with the in-the-moment patterns people employ in their speech and thought as they construct and communicate arguments.

This paper's work will most closely align with this discourse analysis research approach. It borrows Toulmin's idea of a warrant to help analyze a vitally important "in-themoment pattern" in physics students' speech and thought: their epistemological framing of the math at hand. Students' warrants can shift from moment to moment, and these shifts are closely tied to what they interpret as the nature of the math knowledge currently in play.

Practically speaking, this shifting of warrants results in physics students giving different kinds of proof at different times during a mathematical argument. The analysis of the students' mathematical warrants offers a powerful window to describing how they are currently framing their activity.

The idea of different kinds of proof being accepted in an argument is not, in general, a new one. On a grander scale, researchers have noted that what counts as valid proof does not necessarily remain the same as one crosses social or cultural boundaries. One needs look no further than the Creationist or Evolution debate for an example. ${ }^{38}$ On a smaller classroom scale, this phenomenon of shifting justification has also been noted with biology students. ${ }^{39}$

The idea of different kinds of reasoning counting as sufficient proof has also been noted in mathematics education research. Researchers have discussed, for example, the embodied, proceptual, and formal reasons $13+24=24+13.40,41$ The embodied explanation is that adding 24 objects to a collection of 13 objects gives you the same total number as if you started with 13 objects and added 24. A proceptual explanation focuses on how you can manipulate the meaningladen symbols in the problem in a prescribed manner, i.e., you can do the column addition you learned in elementary school and get the same result either way. The formal reason $13+24=24+13$ is that it is assumed true by axiom. It is the commutative property.

The extended case study demonstrates how the flow of a physics problem-solving conversation can be parsed by viewing it as two or more individuals trying to juggle and coordinate various types of mathematical justificationsepistemological framings - in their reasoning.

\section{F. Summary note on terminology}

In summary, we use three terms to describe our epistemological control structure: epistemological framing, epistemological resources, and warrants. Note that by introducing these distinct terms, we are not proposing that these correspond to three distinct cognitive structures. Although that may be the case, we do not at this time have evidence to support that claim. Rather, we use these three terms to provide a way of emphasizing different functional aspects of what may, in the end, turn out to be a reasonably unitary and nonseparable process: the process of making a judgment about what knowledge applies in a particular situation.

The use of the term framing focuses our attention on the interaction between the cue and the response. It stresses that there is an evaluation based on previous general knowledge and experience that is taking place. This evaluation is very often a subconscious one. This paper's focus on the warrants observed in physics students' mathematical arguments is precisely an attempt to define evidence for the inherently implicit cognitive process of framing. True, the warrants themselves are not usually explicitly spelled out in the case study transcripts later in this paper, but the case study will demonstrate that the inferential leap is usually a short easy one (at least relative to simply telling a researcher to look for an "epistemic resource" or a framing).

The use of the term resource focuses our attention on the fact that the kinds of reasons students cite fall into broad categories with a common underlying structure. The use of the term warrant focuses our attention on the epistemic nature of the specific argument being made. For example, a student pointing to a five-line calculation he just performed and a student pointing to an electronic calculator's output may be using slightly different specific warrants (i.e., you can trust a careful manual computation vs you can trust a machine's algorithmic churning). Both students' reasoning, however, falls under the same general class of warrant: algorithmically following a set of established computational steps should lead to a trustable result. We would call this general class of warrant an epistemic resource. It is a general repeatedly observable way students view knowledge. This epistemic resource acts as a cognitive control structure. It is 
one (of many) regulators of students' epistemic framing (i.e., how they implicitly choose to interpret the knowledge at hand).

In the case study that follows, we are somewhat loose with our specific uses of "class of warrant" vs speaking of one particular flavor of warrant specific to the situation at hand. There is simply not always sufficient evidence to carefully distinguish between the two nor is it vital to our general argument (that focusing on warrants gives close-to-thesurface evidence of epistemic framing) to do so.

\section{METHODOLOGY}

Before turning to our extended case study, we give a brief description of the video data used in this study and how our common framings emerged from this data set.

Approximately $150 \mathrm{~h}$ of raw video data of upper-level undergraduate physics students was collected for this study. These students were enrolled in such classes as Quantum Mechanics I and II, Intermediate Mechanics, Intermediate Electricity and Magnetism, and Intermediate Theoretical Methods. Most were physics majors. None was in their first university physics class.

About 80 of these $150 \mathrm{~h}$ (including this paper's case study) come from group homework sessions. Our students would routinely meet outside of class to work on their homework together. We simply would ask their permission at the start of the semester to video tape these meetings. Another $25 \mathrm{~h}$ of the video data set came from individual problemsolving interviews with students. The rest of the video set was made of actual classroom recordings. These classroom videos tended to be less useful for this study since they contained a much smaller amount of student speech.

\section{A. Students' framings are easiest to identify via contrasts and shifts}

Evidence for how students are framing their math use is easiest to pick out when there is some sort of contrast or misunderstanding present. Such framing confusions are common sources of disagreements even in nonphysics settings. ${ }^{42}$ Many mathematical disagreements physics students have with each other reduce to the first student essentially saying "Look at this math issue this way" while the second student is claiming "No, you should be looking at it this other way." The students are debating which aspects of their mathematical knowledge are currently relevant. Examining the warrants ${ }^{34}$ physics students use in their mathematical arguments offers a good window to how they are currently framing their math use.

\section{B. Data selection process}

We needed some sort of selection process that could pare down our $150 \mathrm{~h}$ data set to a collection appropriate for a close careful analysis. The first author was present during $95 \%$ of the tapings themselves and took detailed notes of the students' activity. These notes allowed the video databank to be quickly searched for the best debates, arguments, and misunderstandings. At this early point in the pare-down process, "best" simply meant the debates and arguments that most likely had a lot of material available for possible analysis. Sometimes "best" translated to a simple clock reading. If students spent 5 min arguing about a certain point, there was a good chance a closer analysis might find a relatively large amount of speech that clearly annunciates their ideas. Other times the best arguments were selected for the novelty of their content. An argument about whether an expression simplifies to $x^{2}+2 x+1$ or to $x^{2}-2 x+1$ is likely to be routine. The students are likely to quickly agree on a useful way to resolve the argument. They are likely to share a common framing, which means there would not be much explicit evidence for that framing. However, an argument about a novel issue is much more likely to bring about a variety of approaches, a variety of framings.

The first pass through the $150 \mathrm{~h}$ data set yielded about 50 snippets containing the arguments, debates, and misunderstandings most likely to be explicit and long enough to offer good evidence (i.e., clearly identifiable mathematical warrants) for how the students were framing their math use. Eventually, a framing analysis was carried out on other episodes that did not contain such obvious arguments. Such an extension helped to assure the generality of our framing analysis. The reader is invited to look at the dissertation from which this paper is drawn for such nonargument examples. ${ }^{1(b)}$

Our 50-snippet subset of arguments was meant to offer the best evidence for deciding whether a set of common framings exist and, if they do, what they specifically are. Section III C describes the methodology used to help these common framings emerge.

\section{Knowledge analysis: \\ Common framings emerge from the data set}

In order to make sense of our data, we performed a knowledge analysis., ${ }^{2,43}$ The basic idea is to find a common thread to condense the episodes according to a common analysis scheme. Knowledge analysis is an iterative methodology.

A subset of the 50 sample episodes mentioned above was analyzed individually at first, the goal being to describe what type of warrants the students were using in their mathematical arguments. ${ }^{44}$

Once a small collection of these individual analyses was collected, it became possible to look for consistencies across episodes. Several clusters incorporating similar individual math framing examples were identified. The next step was to do a similar analysis on a new set drawn from those 50 episodes and see if these original clusters could incorporate these new examples of students' mathematical thinking as well. Appropriate changes were made to the clusterings in light of this new data set, and then a third set of episodes was considered. After several iterations, the clustering scheme stopped evolving significantly. Eventually the whole 50 episode subset was used, with each individual episode cycled through more than once.

Four main clusters emerged from this data set's examples of physics students' framing of their math use. They capture 
four general types of justification these students offer for their mathematics: "calculation," "physical mapping," "invoking authority," and "math consistency." These clusterings are not meant to be mutually exclusive or sufficient to span all possibilities. They are merely presented as the most convenient way found of structuring comparisons across many different episodes in our data set.

\section{FRAMING CLUSTERS THAT EMERGED FROM OUR DATA SET}

Our knowledge analysis led us to classify the student interactions into four common framing clusters: calculation, physical mapping, invoking authority, and math consistency. These framings parallel the discussion of $x_{f}=x_{i}+\langle v\rangle \Delta t$ in Sec. I. We begin with a brief overview of each and then present a more complete discussion and comparison of the four clusters. Finally, we discuss inter-rater reliability.

\section{A. Framing 1: Calculation}

A calculation framing, like all the other framings that emerged from the data set, is primarily identified by the general class of warrant students choose to use. In this case the epistemological resource (i.e., the general class of warrant observed) is algorithmically following a set of established computational steps should lead to a trustable result. The specific warrants used, like all the other warrants we identified in our data set, couple closely to the epistemological resources currently activated by the student. Epistemological resources, recall, are control structures. They lead the student to frame the knowledge at hand in a certain way, which focuses the student's attention on a particular subset of his total knowledge.

In a calculation framing, students rely on computational correctness. The warrant may be implicit, especially in nonargumentative settings. If, say, an instructor was deriving $y_{f}=y_{i}+v_{i} t-\frac{1}{2} g t^{2}$ from $d^{2} y / d t^{2}=-g$, she would probably just carefully explain her steps to her students. They would likely accept the result without further thought. It is rare to explicitly explain, "OK, because carefully following a set of computational steps allows one to trust a result, we should trust this derivation." It would rely on an unspoken epistemological resource, one that is shared because both instructor and student frame the discussion as calculation.

\section{B. Framing 2: Physical mapping}

When physics students frame their math use as physical mapping, they support their arguments by pointing to the quality of fit between their mathematics and their intuition about the physical or geometrical situation at hand. This class of warrant can be associated with the epistemological resource: a mathematical symbolic representation faithfully characterizes some feature of the physical or geometric system it is intended to represent. Again, it is through identifying these (relatively close-to-the-surface) warrants that a researcher can get information about the (relatively implicit) epistemological framing process in the student's mind.
For example, suppose we wanted to explain why the expression for the force exerted by a spring, $F=-k x$, includes a negative. We might explain how stretching a spring makes it pull backward as it tries to contract back to its natural length. If you compress the spring, it will push back against the compression as it tries to expand. In both cases the spring force is opposite the way the spring is deformed. That is, if $k x$ is positive (say you extend the spring to the right) then the spring pulls in the negative (i.e., left) direction. If $k x$ is negative (say you compress the spring leftward) the spring exerts a force to the right (positive) direction. This kind of mechanistic chaining of reasons is a common characteristic of this framing. Again, we do not necessarily have to spell out an explicit warrant or our math-should-model-the-world epistemological resource. They come along with a physical mapping framing.

There is a more general point about distinguishing a calculation framing from a physical mapping framing. At some level, all mathematics is ultimately grounded in physical experience. A child learns to associate " 1 " with a single object, " 2 " with a collection of two objects, and so on. Higher and higher mathematics are built up by analogy and extension of what are ultimately physically grounded ideas. ${ }^{45,46}$ The distinction between a calculation framing and a physical mapping framing largely concerns a person's in-the-moment awareness of the physical referents of her math.

We note that for this work, we do not distinguish between the use of physical statements from the use of abstract geometrical statements as warrants. This is because in the examples we observed, the geometry arose out of the location of the physical situation in three-space. We expect that if a wider class of situations was considered, it might be appropriate to separate physical and geometrical framings.

\section{Framing 3: Invoking authority}

Suppose we were trying to convince you what the rotational inertia of a solid sphere was. We might simply pick up an introductory physics book, thumb through the index until we found "rotational inertia," turn to page 253 , and point at an entry in a table that says "solid sphere, $I=\frac{2}{5} M R^{2}$." Perhaps you would accept our argument also accepting the implicit class of warrants (i.e., epistemological resource) that underlies our reasoning: information that comes from an authoritative source can be trusted.

An invoking authority framing is often closely tied to finding the right level of detail to go into during a problem. It is unreasonable to take every single problem down to absolute first principles every time. Some results will always simply be taken for granted. Perhaps you would be more likely to accept our earlier argument for the rotational inertia of a solid sphere if we were engaged with a larger problem such as finding the time it would take such a sphere to roll down a given ramp. You might judge the specific value of the sphere's rotational inertia to be sufficiently irrelevant to the problem's main purpose to permit us to quote from the textbook.

Another common trait of the invoking authority framing is the absence of extended chains of reasoning. "Chaining" 
TABLE I. Four common framings and their primary (i.e., warrants) and secondary indicators.

\begin{tabular}{|c|c|c|c|c|}
\hline & Calculation & Physical mapping & Invoking authority & Math consistency \\
\hline Class of warrant used & $\begin{array}{l}\text { Correctly } \\
\text { following } \\
\text { algorithmic } \\
\text { steps gives a } \\
\text { trustable result }\end{array}$ & $\begin{array}{l}\text { Goodness of fit } \\
\text { between mathematical } \\
\text { and physical } \\
\text { observations or } \\
\text { expectations } \\
\text { attests to a } \\
\text { result }\end{array}$ & $\begin{array}{l}\text { Authoritatively } \\
\text { asserting a result } \\
\text { or a rule gives it } \\
\text { credence }\end{array}$ & $\begin{array}{l}\text { Similarity or } \\
\text { logical } \\
\text { connection to } \\
\text { another math } \\
\text { idea offers } \\
\text { validation }\end{array}$ \\
\hline \multirow[t]{3}{*}{ Other common indicators } & $\begin{array}{l}\text { Focus on } \\
\text { technical } \\
\text { correctness }\end{array}$ & $\begin{array}{l}\text { Often aided by } \\
\text { a diagram }\end{array}$ & Quoting a rule & $\begin{array}{l}\text { Analogy with } \\
\text { another math } \\
\text { idea }\end{array}$ \\
\hline & $\begin{array}{l}\text { Math chaining: } \\
\text { need this to } \\
\text { get that }\end{array}$ & $\begin{array}{l}\text { Demonstrative } \\
\text { gesturing }\end{array}$ & $\begin{array}{l}\text { Absence of } \\
\text { mechanistic } \\
\text { chaining }\end{array}$ & Categorization \\
\hline & & $\begin{array}{l}\text { Mechanistic } \\
\text { chaining }\end{array}$ & $\begin{array}{l}\text { Little } \\
\text { acknowledgment } \\
\text { of substructure }\end{array}$ & \\
\hline
\end{tabular}

has been closely tied to students' mechanistic reasoning. ${ }^{47,48}$ When a student links together a series of implications, she is chaining. An example would be "adding another resistor in series puts another obstacle in the current's way, so the total resistance goes up, but the battery's push remains the same, so the current flowing decreases." Students engage in mathematical chaining arguments as well. The calculation framing often cues reasoning like " $A=B C$, but we do not know $C$, but we can use $C=E F$ to get $C$, then we can use $C$ to get $A$." The electric current example just above could be a nice example of chaining while in a physical mapping framing if the student was simultaneously thinking about the formula $\Delta V=I R$. Chaining is usually absent or severely limited if the student is framing his math use solely as invoking authority, but it is often present in both the calculational and physical mapping framings.

\section{Framing 4: Mathematical consistency}

Suppose you were trying to explain Coulomb's law for the electric force, $\vec{F}_{e}=\left(1 / 4 \pi \varepsilon_{o}\right)\left(q_{1} q_{2} / r^{2}\right) \hat{r}$, to a student. You might remind him of the expression for the gravitational force, $\vec{F}_{g}=-\left(G m_{1} m_{2} / r^{2}\right) \hat{r}$, and demonstrate how ideas from this more familiar bit of math map to Coulomb's law. Both forces depend on the relative strengths (mass or charge) of the two objects in question. Both forces fall off with respect to distance in the same way and both include a proportionality constant $\left(G\right.$ or $\left.1 / 4 \pi \varepsilon_{o}\right)$ that must be experimentally measured. Even disanalogous observations can be illuminating. Gravity is always attractive; hence the negative sign is explicitly included in front of the always positive masses. An electric force can be attractive or repulsive, so the implicit signs on the positive or negative charges, $q_{1}$ and $q_{2}$, will determine the direction of the Coulomb force.

Implicit in your discussion with the student would be the class of warrants indicative of a math consistency framing: mathematics and mathematical manipulations have a regu- larity and reliability and are consistent across different situations. Establishing a common underlying mathematical structure allows one to trust the relevant set of relations and inferences.

\section{E. Correlates of the four framing clusters}

Our four common framings are primarily identified via the warrants physics students use in their mathematical reasoning. Other indicators, however, have been observed to cluster preferentially around certain framings. Table I summarizes these primary (i.e., warrants) and secondary framing indicators we have observed in our data set.

Framing is a dynamic cognitive process. A person's mind makes an initial judgment regarding the nature of the situation at hand, but that judgment is continually updated and reevaluated. New information comes to the student all the time whether in the form of a classmate's comment, an interviewer's interjection, simply turning to a different page in a textbook, or even spontaneous random associations within her own brain. This new information can lead a student to reframe her activity. As a result, the epistemological framings observed in this students' work can extend over a range of time periods. We have found examples in our data set ranging from $10 \mathrm{~s}$ to $10 \mathrm{~min}$.

\section{F. Statement of this study's purpose}

We now have laid a sufficient foundation upon which to state this paper's main premise in its full detail: framing and epistemic resources are, by definition, cognitive modeling elements. They aim to capture an important aspect of students' thinking (i.e., its tendency to selective attention), but one can never directly observe, say, an epistemic resource. We suggest that analyzing the warrants students rely on provides a way to pin down evidence of their framing and the epistemic resources activated in their minds. 
Why have we put "class of warrant used" on a pedestal in Table I while relegating various student actions to "other common indicators"? Is not an action like "quoting a rule" also excellent evidence of an invoking authority framing? It certainly is, but the phase space of possible student actions is immense. Consider one student who confidently states and restates some canned phrase (maybe "work is path independent") and another student who combs through 50 pages of his textbook looking for the formula $I=\left(\frac{2}{5}\right) M R^{2}$. Their actions are different, but the class of warrant that underlies their action tends to be the same: authoritatively asserting a rule or result gives it credence. Both students are framing their work as invoking authority. The dimensionality of the warrant phase space is much lower (on the order of 4) as the upcoming case study demonstrates.

\section{G. Inter-rater reliability of epistemological framing analysis}

The value of this study's epistemological framing analysis depends in part on how readily other researchers can apply it consistently. An inter-rater reliability study was carried out by giving this paper's methodology discussion to another researcher and that researcher was then asked to parse a new transcript for epistemological framing (i.e., identify the class of warrants underlying the students' arguments). Details of this inter-rater reliability test are given in Chap. 4 of the first author's dissertation. ${ }^{1(b)}$ Different researchers agreed on their warrant codes (and hence their framing codes) $70 \%$ of the time for a novel transcript before any consultation or discussion. This figure improved to $80 \%$ after discussion. As our case study demonstrates, this warrant analysis does indeed parse students' thought into frames in a natural reasonable way.

We do not expect our warrant coding scheme to yield a $100 \%$ consistent coding of a random transcript. Students' thinking is simply not that cleanly compartmentalized. Indeed, we argue [see Chap. 7 in Ref. 1(b)] that one characteristic of expertise in physics problem solving is the ability to effectively blend these four framings dynamically.

Two issues are relevant here. First, there is the question of how often students are observed to spend an appreciable time, say a minute or more, uniquely in one of this paper's four common framings. Of all the data analyzed for this study, perhaps less than $50 \%$ can be cleanly coded in minuteor-longer chunks under one of these general framings.

The second notion of "clean coding" of framing that is relevant concerns not these minute-long pure state framings but rather our ability to identify smaller chunks in hybrid framings. Calculation, physical mapping, invoking authority, and math consistency do a reasonable job of spanning the space of these students' mathematical arguments. We observed that about $90 \%$ of a random episode or more can be seen as made up of behavior indicative of those four landmark framings. But at this stage of student development (upper-division physics majors) hybrids are common. Perhaps a student quotes a few computational rules as he performs a long calculation. Maybe a student makes an analogy to both a similar physical situation and a similar math structure. As the inter-rater reliability test shows, researchers can

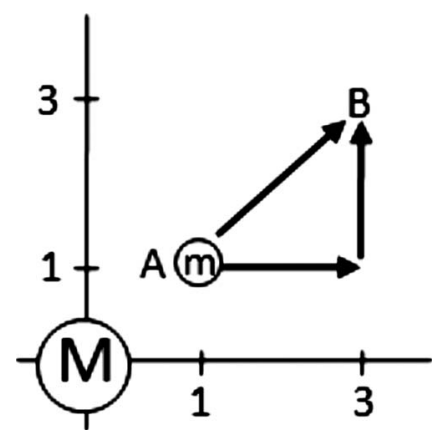

FIG. 1. Case study problem.

still use this study's analysis scheme to identify evidence of these elemental framings in a piece of transcript that is, in general, a more complicated hybrid framing.

\section{CASE STUDY}

We now turn to our case study. This detailed example illustrates how this paper's warrant-based framing analysis can be applied to parse an authentic conversation among physics students.

The students' framing of their math use plays a significant role in the episode. The principal dynamic concerns how to interpret the math at hand. A significant amount of these students' energy goes into trying to establish the epistemological framing they see as appropriate.

Their thinking is dynamic. Different bits of their mathematical knowledge are activated and deactivated as they frame and reframe their activity. Sometimes framing differences have marked effects. The students sometimes talk past each other, neither one seeming to hear what the other is saying, because they are framing their work differently. Sometimes a student's framing can exhibit considerable resistance to change. As students become more sophisticated and expertlike, we conjecture that students become more flexible in their framing and increasingly create hybrid frames. ${ }^{49}$

Our case study comes from a group of three students enrolled in the class Intermediate Theoretical Methods (PHYS 374). One is a junior (S2) and the other two are sophomores (S1 and S3). These three students met regularly outside of class to work on their homework together, and this episode was taped during one such homework session.

\section{A. Question}

Our episode starts in the middle of their work on one of that week's homework problems. The problem they were considering reads:

A rocket (mass $m$ ) is taken from a point $(A)$ near an asteroid (mass $M$ ) to another point $(B)$. We will consider two (unrealistic) paths as shown in the Fig. 1. Calculate the work done by the asteroid on the rocket along each path. Use the full form of Newton's universal law of gravitation (not the flat earth approximation $m g$ ). Calculate the work done by using the fundamental definition of work: $W_{A \rightarrow B}=\int_{A}^{B} \vec{F} \cdot d \vec{r}$. 
The reader familiar with the physics of this example will recognize an attempt to get the student to see how the theorem that says potential energy is path independent arises out of explicit calculation. Not all of the students in this discussion recognized the point of the problem from the beginning.

\section{B. First framing clash}

During this episode, the students are trying to decide if the work done should be the same along the two paths from $A$ to $B$. They had previously suppressed the $G, m$, and $M$ constants and written the (incorrect) $)^{50}$ equation $\int_{\sqrt{2}}^{3 \sqrt{2}}\left(1 / r^{2}\right) d r=\int_{1}^{3}\left[1 /\left(y^{2}+9\right)\right] d y+\int_{1}^{3}\left[1 /\left(x^{2}+1\right)\right] d x \quad$ on the blackboard to express the work done along the direct and two-part paths, respectively. They have also copied the diagram of the situation from the problem statement.

The students are standing at the blackboard where all the relevant equations and diagrams appear. We focus on the type of justification each student offers for his math arguments:

1. S1: what's the problem?

2. You should get a different answer

3. from here for this [points to each path on two-path diagram $\underset{\longrightarrow}{\longrightarrow}]$

4. S2: No no no

5. S1: They should be equal?

6. S2: They should be equal

7. S1: Why should they be equal?

8. This path is longer if you think about it [points to two-part path again].

9. S2: Because force, err, because

10. work is path independent.

11. S1: This path is longer, so it should have [points to two-part path again],

12. this number should be bigger than

13. S2: Work is path independent. If you

14. go from point $A$ to point $B$,

15. doesn't matter how you get there,

16. it should take the same amount of work.

Lines 1-6 contain the main issue of this episode. While S1 thinks there should be different amounts of work done on the small mass along the two different paths, S2 believes the work done should be the same.

In the language of formal argumentation theory, ${ }^{34} \mathrm{~S} 1$ makes the claim that more work is done along the two-part path, and he offers the data that the two-part path is longer. An unspoken warrant exists that connects his data to his claim: the particular mathematics being used should align with the physical systems under study. The goodness of fit between the math at hand and the physical system attests to the validity of one's conclusions. The work formula given, $W_{A \rightarrow B}=\int_{A}^{B} \vec{F} \cdot d \vec{r}$, seems to say "force times distance" to $\mathrm{S} 1$. The two-part path has more "distance," and S1 thus draws justification for his answer.

S1's warrant thus suggests he is framing his activity as physical mapping. His use of a diagram in lines $1-3,7$, and 8 supports this characterization. He gestures to the different paths as he points out that the two-part path is physically longer. Use of a diagram as intermediary between the physical situation and the mathematics is a commonly observed indicator of a physical mapping framing. A diagram can often help a student entwine physical and mathematical ideas as he examines how the two fit together.

$\mathrm{S} 2$ not only has a different answer than S1 but he is also framing his use of mathematics in a different way. S2 claims that the work done on the small mass should be the same along the two paths "because work is path independent" (lines 9 and 10). His data are a familiar mantra (though he omits mentioning how this statement is only valid for conservative forces such as gravity). The unspoken warrant that S2 is relying on concerns the common use of rules and definitions in math and physics: sometimes previous results are simply taken as givens for speed and convenience. S2 is framing his math use as invoking authority.

After hearing S2's counterargument, S1 repeats himself. In lines 11 and 12, he restates his longer-path justification and again points to the relevant features of the diagram they had previously drawn on the board. S2 responds by restating work is path independent in line 13 and again, slightly differently, in lines 14-16.

The most important observation in this first clip is that S1 and S2 are disagreeing over much more than merely the answer itself. Explicitly, they are debating whether or not more work is done along the longer path. Implicitly, they are arguing over the most useful way to frame their present use of mathematics. S1 never explicitly says "Please respond to my claim in a way that maps our math to some detail of the physical situation I may have overlooked." His phrasing and gesturing in his initial argument (lines 7 and 8) and beyond (lines 11 and 12) imply this framing request, though.

When $\mathrm{S} 2$ responds with his rule citation, he is not merely arguing for a different answer. He is pushing for a different type of warrant for judging the validity of a given answer. S2's invoking an authority framing may have even prevented him from really hearing what S1 was saying. S1's framing request may have passed by $\mathrm{S} 2$ unnoticed because he was too caught up in the subset of all his math resources that his invoking authority framing had activated within his mind. At any rate, $\mathrm{S} 2$ responds in lines 9 and 10 with a different type of justification than what S1 was expecting.

When $\mathrm{S} 1$ repeats himself in lines 11 and 12, he is implicitly repeating his bid for a physical mapping framing. One can imagine a situation when S2's invoking authority justification would simply be accepted without incident, but here it did not align with S1's present framing. S2 does not respond to this reframing request and repeats his answer as he remains in invoking authority.

There is thus an intense framing argument going on under the surface of this debate. Sensing that he is not making any headway in the framing battle, S1 now moves to shift both himself and S2 into a third framing.

\section{Temporary agreement on a third framing}

S1 now makes a move toward a third way of addressing the mathematics at hand. He suggests they churn away and calculate what numeric answers their expressions actually 
give (which happens during lines 31-41). S2 accepts for a time.

17. S1: OK, that's assuming Pythagorean

18. Theorem and everything else add[s].

19. Well, OK, well is this-what was the

20. answer to this right here [points to

$\left.\int_{\sqrt{2}}^{3 \sqrt{2}}\left(1 / r^{2}\right) d r=\int_{1}^{3}\left[1 /\left(y^{2}+9\right)\right] d y+\int_{1}^{3}\left[1 /\left(x^{2}+1\right)\right] d x\right]$ ?

21. What was that answer?

22. S2: Yeah, solve each integral numerically.

23. S1: Yeah, what was that answer?

24. S3: Each individual one?

25. S1: Yeah, what was

26. S3: OK, let me, uhh [S3 starts typing into MATHEMATICA]

27. S1: Cause path two is longer than path one, so

28. S2: May I, for a minute [S2 writes on a small corner of the blackboard, but never speaks about what he writes]?

29. S1: and path one was this.

30. S2: Gimme this, I wanna think about something.

31. S1: Just add those up, tell me the number for this [points to integrals again]

32. and I'll compare it to the number of

33. S3: OK, the $y$ one is 0.15 .

34. S1: I, just give me the, just sum those up.

35. I just want the whole total.

36. I just want this total quantity there,

37. just the total answer [points to integrals again].

38. S2: Oh, it was 0.4

39. S3: No, that's the other one [direct path].

40. S1: you gave it to me before, I just didn't write it down.

41. S3: Oh I see, point, what, 0.618

42. S1: See, 0.618, which is what I said,

43. the work done here should be larger

44. than the work done here 'cause the path [points to two-path diagram]

45. S2: No, no no, no no no

46. S3: the path where the $x$ is changing

47. S2: Work is path independent.

48. S1: How is it path independent?

49. S2: by definition

50. S3: Somebody apparently proved this before we did.

S1 attempts to reframe the discussion in lines 19-21. He points to the integrals they've written and asks, "Well, OK... what was the answer to this right here? What was that answer?" He is calling for someone to evaluate each of their expressions for the work so that he can compare the numeric results. This argument relies on another kind of warrant. Mathematics provides one with a standardized selfconsistent set of manipulations and transformations. Performing a calculation or having a computer do it for you according to these rules will give a valid trustable result. S1 is moving to reframe their math use as calculation.

Even though S1 does not explicitly detail the new warrant he is proposing, S2 quickly zeroes in on it. He immediately responds "yeah, solve each integral numerically" (line 22). Compare this successful fluid epistemic frame negotiation with the struggle of the previous snippet. Lines 1-16 had S1 pushing for physical mapping while S2 lobbied for invoking authority. Both stuck to their positions, resulting in an inefficient conversation. Neither was accepting what the other was trying to say. Lines 19-22 have S1 and S2 agreeing, for the moment, on what type of mathematical justification should count.

With the calculation frame having been negotiated, lines 23-41 are mostly about S1 directing S3 to input the proper expressions into MATHEMATICA, a common software calculator package. They finish with MATHEMATICA in line 41. It turns out that the radial path integral, $\int_{\sqrt{2}}^{3 \sqrt{2}}\left(1 / r^{2}\right) d r$, is equal to 0.47 , while the two-part path integrals, $\int_{1}^{3}\left[1 /\left(y^{2}+9\right)\right] d y+\int_{1}^{3}\left[1 /\left(x^{2}+1\right)\right] d x$, evaluate to 0.618 . S2 was correct back in lines $1-16$. The same amount of work should be done along the two paths. While the radial integral is correct as written (within a negative sign), they have neglected the cosine term from the dot product $\vec{F} \cdot d \vec{r}$ in the two-part path integrals.

S1 takes the result of their calculation argument to support his earlier physical mapping framing: "see, 0.618, which is what I said, the work done here should be larger than the work done here "cause the path..." (lines 42-44). This move is quite impressive. Here, $\mathrm{S} 1$ is using his calculation framing as a subroutine of sorts. He is nesting his computation within a larger scheme of supporting his physical mapping argument of longer path means more work.

$\mathrm{S} 1$ gives another hint that the physical mapping framing has not completely decayed while they are calculating. In the midst of the MATHEMATICA work, he tosses in "cause path two is longer than path one" (line 27). This example illustrates the "hybrid" point made in the inter-rater reliability section of this paper. Physics students' thinking is simply not always compartmentalized. The four framings only represent general clusters of reasoning. That $\mathrm{S} 1$ tosses in a still-active piece from his previous physical mapping into the calculation is neither an anomaly of thought nor a failure of this paper's framework. A likely mark of expertise in physics is a more expansive framing that allows students to access and interact the resources in the framings described here. ${ }^{49}$ Indeed, this problem was set up and assigned for the very purpose of encouraging students to look for coherency among various framings like S1 is doing here.

Earlier, we claimed that less than $50 \%$ of a random episode of student thinking could be cleanly coded as an elemental form of calculation, physical mapping, invoking authority, or math consistency. Still, we claimed that about $90 \%$ of a transcript could be seen as a molecular combination of overlapping bits of them. Lines 19-41 are an example that is mostly calculation but is fuzzed somewhat by physical mapping.

$\mathrm{S} 2$ responds in a familiar way to $\mathrm{S} 1$ 's recall of physical mapping in lines 42-44: "see, 0.618, which is what I said, the work done here should be larger than the work done here "cause the path." S2 returns to invoking authority to justify his equal-work assertion in lines 45 and 47. "No, no no, no no no... work is path independent." When S1 presses him for more detail, "how is it path independent?" (line 48), S2 and S3 respond "by definition" (line 49) and "somebody apparently proved this before we did" (line 50). 


\section{Even stronger bid for physical mapping}

The replies of S2 and S3 in lines 49 and 50 do not contain the type of justification S1 seeks. The next block of transcript begins with $\mathrm{S} 1$ making another strong bid for physical mapping.

51. S1: OK, I don't understand the concept then,

52. because you're saying it's path independent.

53. S2: I'm saying, if you're at the bottom of a hill

54. S1: all right

55. S2: and you want to drive to the top of the hill

56. S1: right

57. S2: and there's a road that goes like this,

58. a road that goes like this, and a road that's like this [draws ? ? ],

59. it takes the same amount of energy to get

60 . from the bottom to the top.

61. It doesn't matter which one you take.

62. S1: OK, then you tell me this then;

63. work is force times distance, right?

64. S2: It's the integral of $f d r \ldots f d r$, yeah.

65. S1: So if you're going this $r$, and [draws

66 . you're going this $R$, which one has more work?

67. S2: If there's constant force?

68. S1: Constant force on each on

69. S2: This one if it's the same force [points to long $R$ path].

70. S1: OK, now the same force is acting on that

71. S2: No. No no. Because this one [radial] has

72. direct force the whole time.

73. See, there's lesser force [gestures at two-path diagram].

74. S1: OK

75. S2: in each one of these [two part path]

76. S1: OK. All right

77. S2: your forces are

78. S1: I see what you mean. I see what you mean.

79. Here we're taking

80. S2: Here we're supposed

81. to be compensating for that

82. S1: We're just taking the $x$ component [gestures at two-path diagram]

83. of the force here, and the $y$ component

84. of the force there. You're probably right.

85. You've probably been right the whole time

86. Are we thinking about that correctly then?

87. I agree with what you're saying.

S1 begins this last transcript chunk with another bid to frame their math use as physical mapping. "I don't understand the concept then, because you're saying it's path independent" (lines 51 and 52).

S2 responds to this newest bid with an interesting hybrid of his own. He is still quoting "work is path independent" but he now couches that rule in terms of a physical situation. He draws a picture of various paths up a hill and asserts "it takes the same amount of energy to get from the bottom to the top. It doesn't matter which one you take" (lines 53-61).

S2's latest response still partly reflects an invoking authority framing because it offers no physical mechanism for why the work done by gravity should be the same along any of the paths up the hill. Technically, your car would burn more gasoline along the curviest path, but S2 does not acknowledge this point and may not have even considered it in light of the inertia invoking authority is exhibiting in his thought. Perhaps S2 has a more detailed physical mechanism in his mind, but he does not articulate it here.

Nonetheless, S1 recognizes a glimmer of the type of justification he seeks in S2's latest argument. S1 presses further on the longer-path issue. "OK, then you tell me this then; work is force times distance, right?... So if you're going this $r$, and you're going this $R$, which one has more work?" (lines 62-66). This question is S1's most explicit call yet for a physical mapping framing. He closely juxtaposes a mathematical point (work is force times distance) and a diagramaided observation of a longer path (his $r$ and $R$ picture).

This reframing bid tips $\mathrm{S} 2$. His hint of a physical mapping framing in lines 57-61 asserts itself, putting him in a much better position to understand S1's argument. For the first time in this conversation, $\mathrm{S} 2$ explicitly addresses a physical detail relevant to the physical mapping S1 is attempting: "if there's constant force?" (line 67) S1 quickly affirms that assumption and $\mathrm{S} 2$ correctly concludes that more work would be done on the long $R$ path. When $\mathrm{S} 1$ quickly moves from this hypothetical $r$ and $R$ case back to the homework problem (line 70), S2 immediately points out the inconsistency. "No. No no. Because this one [radial] has a direct force the whole time. See, there's lesser force... in each one of these [twopart path]... here we're supposed to be compensating for that" (lines $71-75,80$, and 81). S2 gestures to the problem's diagram during this physical mapping. The gravitational force vector and the displacement vector are (anti)parallel for the radial path, hence you need to consider the full magnitude of the gravitational force in calculating the work done along that path. These two vectors do not align perfectly along the two-part path; hence you only consider a component of the force there.

S1 quickly accepts and confirms this argument (lines 7887 ), which is the first fully articulated physical mapping argument S2 had offered during this conversation. His quick comprehension and acceptance occur because S2 has now framed their problem solving in the way S1 has. S1 was mentally ready to accept such an argument.

S2's reluctance to adopt a physical mapping framing implies an activation failure, not lack of sophistication or naivety. His reluctance was certainly not due to simple inability. He was, after all, the one who actually wrote the integrals (which do not contain the necessary cosine factors but, according to S2, were meant to reflect the "lesser force" idea) in the minutes leading up to the presented transcript. S2 quickly generated a physical mapping argument once he framed the discussion as physical mapping, i.e., once he activated the relevant subset of his mental resources.

\section{E. Summary of the case study}

This case study illustrates how epistemological framing negotiation and communication can be a powerful dynamic in physics students' work. S1 and S2 disagreed over much more than whether the gravitational work done was independent of path. Their disagreement over what type of justification was appropriate drove this conversation. Much of this debate was implicit. S1 never came out and said, for ex- 
ample, "please respond to me in a way that points out some detail of the physical situation that I have not mapped correctly to the mathematics we're using." The epistemological framing analysis presented in this paper offers a way of making this implicit conversation dynamic explicit to teachers and physics education researchers.

In this case study these epistemological framings had considerable inertia. S2 remained in invoking authority despite several prods. S1's commitment to physical mapping allowed those prods to keep happening. Other case studies show students shifting frames much more readily. ${ }^{1(\mathrm{~b})}$

\section{F. Other examples}

While we believe that our case study presents a compelling case for the value of our epistemological framing via warrants analysis, we also note that there are examples in the published literature that demonstrate the "stickiness" of epistemological framing and would lend themselves well to an analysis of the type described here.

In a previous paper by the authors ${ }^{51}$ we discuss a case study in which a group of students solving a problem in quantum mechanics is "stuck" for nearly $15 \mathrm{~min}$ in a computational or mathematical-consistency frame unable to detect an error in the setup of their equations. Only when the group shifts to a physical reasoning frame does the error become clear.

In a paper describing students' functional epistemology in introductory algebra-based physics, Tuminaro and Redish cited an example of a student so stuck in a mathematicalconsistency frame that she fails to notice that her statement that her dormitory room has a volume of $1 \mathrm{~m}^{3}$ is absurd..$^{52}$

\section{CONCLUSION AND IMPLICATIONS}

\section{A. Summary}

In this paper we have argued that analyzing student problem solving from the point of view of the kind of warrants (or epistemological resources) they choose to use gives insight into the way the student is framing the mathematical task at hand. From a large number of ethnographic observations of students in upper-division physics classes we selected situations in which students were taking contrasting views on the approach to be used. From these salient data we created a classification of warrants that we believe indicate the students' epistemological framing of the task.

The students in our case study disagree over much more than an answer. They each frame their activity differently and hence try to apply a different type of warrant to judge the validity of their claims. The students exert various pushes and pulls on each other as they try to negotiate a common epistemological framing. Vary rarely are these reframing bids explicit. Nonetheless, these framing debates underlie the speech in both of our case studies. When a common framing is established, the conversation tends to be richer and more efficient. The warrant analysis presented here is meant as a useful tool for finding evidence of what is, at its root, an implicit cognitive process.

\section{B. Implications for instruction}

Our analysis has important implications for teachers as well as for researchers. First, being aware of framing can help an instructor be aware of when he and his students are not communicating — when they are "not on the same page." Second, being aware of framing can lead an instructor to understand the value of hybrid and flexible framing and lead to her evaluating student progress in a fashion that is both subtler and more productive. We elaborate briefly on each of these points and speculate on how an instructor might respond to these issues effectively.

\section{Being aware of framing can reveal failures in communication}

Framing differences like those in the case study here, and the miscommunications that accompany them, could certainly occur between instructor and student as well as between students. Both the instructor and students will naturally frame what occurs during a lesson, but there is no guarantee they will frame each part of the lesson in the same way. A teacher may calculate for a while and then want to make a point about how an equation matches a physical expectation. The teacher may even offer a signal that he's switching approaches, but perhaps that signal is not sufficient to tip the students. They may merely try to interpret his physical mapping comments through a calculation lens or even reject the physical mapping reframing as irrelevant and stop paying attention. Perhaps a professor gives an extended math consistency discussion, carefully explaining how the math at hand is analogous to a more familiar math idea. Maybe his students are framing the discussion as invoking authority and instead hear a series of math facts to be accepted on faith.

We conjecture that there are (at least) two ways to combat such teacher or student framing misunderstandings. The first is for a teacher to simply exaggerate her framing cues. If the situation calls for conveniently quoting a rule, spend a little extra time explaining your reasons for doing so. If it is obvious to you, as a teacher, that a physical mapping discussion is in order, make that (and your reasons for believing so) more explicit to your class. More explicit framing cues might lessen the probability of miscommunication due to a framing mismatch.

A second antidote to teacher or student framing mismatches is for the teacher to gather more evidence, in real time, of her students' framing. In a traditional lecture, information tends to only flow from the professor to the students. Such a lecturer will have scant evidence available for how her students are framing the lesson. Asking questions that have simple phraselike answers may give a teacher evidence of the simple correctness or incorrectness of the class's answers but is only of marginal help for inferring the students' epistemological framing. Engaging one's students in extended discussions during class is the best way to get valuable framing evidence. Asking open-ended questions that give students a wide range of possible responses will require them to explain their reasoning to a much greater depth. As they explain their justifications for their claims, their framing will become much more apparent to the teacher. Framing 
mismatches will become much easier to diagnose in real time.

\section{Being aware of framing can help an instructor better diagnose the cause of students' difficulties}

As instructors, we all have experience of students who appear oblivious to a course of action or a solution method that seems utterly obvious to us. The simplest explanation for such a phenomenon can sometimes be that those students just do not have the requisite knowledge or experience that the problem demands.

However, if an instructor is aware of the fact that students may "get stuck" in a framing that limits their access to tools and knowledge they may not only possess but be good at, the instructor will have an alternate explanation of the difficulty the students may be experiencing. That instructor may be less likely to "write off" his students as incompetent and more likely to try prodding them into a different framing. Developing homework questions comes to be seen as creating tasks of sufficient richness and complexity to help students develop these frame-juggling skills on their own. This issue is discussed in more detail in Chap. 7 in Ref. 1(b).

\section{Implications for future research}

This analysis opens possibilities for significant research efforts by illuminating a dimension of student performance that is rarely considered as a component of "student difficulties" but that potentially plays a critical and controlling role for many students. Much more work is needed, both in improving the methodology of identifying student framing and in explicating the role framing difficulties play in the typical classroom. Another interesting question is how (if at all) physics expertise differs from novice problem-solving behavior when viewed through a framing analysis lens. We conjecture that experts are more likely to both create broader framings that blend more knowledge and skills and to explicitly search for coherence between different framings, but this remains an open research question.

\section{ACKNOWLEDGMENTS}

We gratefully acknowledge the support and suggestions of many members of the University of Maryland Physics Education Research Group and of visitors to the group including David Hammer, Andrew Elby, Rachel Scherr, Rosemary Russ, Ayush Gupta, Brian Frank, Saalih Allie, and Steve Kanim. This material is based on work supported by the U.S. National Science Foundation, including Grants No. REC 0440113 and No. DUE 05-24987.
${ }^{1}$ This paper is a detailed extension of a short conference proceeding paper (a) T. J. Bing and E. F. Redish, in Proceedings of the 2008 Physics Education Research Conference, edited by C. Henderson, M. Sabella, and L. Hsu, AIP Conf. Proc. No. 1064 (AIP, New York, 2008), p. 71; it is based on (b) T. J. Bing, Ph.D. thesis, University of Maryland, 2008, http:// www.physics.umd.edu/perg/dissertations/Bing/

${ }^{2}$ B. Sherin, How students understand physics equations, Cogn. Instruct. 19, 479 (2001).

${ }^{3}$ A. A. diSessa, Toward an epistemology of physics, Cogn. Instruct. 10, 105 (1993).

${ }^{4}$ A. A. diSessa and B. Sherin, What changes in conceptual change?, Int. J. Sci. Educ. 20, 1155 (1998).

${ }^{5}$ D. Hammer, Student resources for introductory physics, Am. J. Phys. 68, S52 (2000).

${ }^{6}$ E. F. Redish, in Proceedings of the International School of Physics, "Enrico Fermi" Course CLVI, edited by E. F. Redish and M. Vicentini (IOS, Amsterdam, 2004), p. 1.

${ }^{7}$ D. Hammer, A. Elby, R. E. Scherr, and E. F. Redish, in Transfer of Learning: From a Modern Multidisciplinary Perspective, edited by J. P. Mestre (Information Age, Greenwich, CT, 2005), p. 89.

${ }^{8}$ E. F. Redish and K. A. Smith, Looking beyond content: Skill development for engineers, J. Eng. Educ. 97, 295 (2008).

${ }^{9}$ A. D. Redish, S. Jensen, and A. Johnson, A unified framework for addiction: Vulnerabilities in the decision process, Behav. Brain Sci. 31, 415 (2008).

${ }^{10}$ D. O. Hebb, The Organization of Behavior (Wiley, New York, 1949).

${ }^{11}$ M. Wittmann and R. E. Scherr, in Proceedings of the 2002 Phys- ics Education Research Conference, edited by S. Franklin, J. Marx, and K. Cummings (AIP, New York, 2002).

${ }^{12}$ D. Hammer and A. Elby, in Personal Epistemology: The Psychology of Beliefs about Knowledge and Knowing, edited by B. Hofer and P. Pintrich (Erlbaum, Mahwah, NJ, 2002), p. 169.

${ }^{13}$ D. Hammer, in Proceedings of the International School of Physics, "Enrico Fermi" Course CLVI, edited by E. F. Redish and M. Vicentini (IOS, Amsterdam, 2004), p. 321.

${ }^{14}$ D. Hammer, Epistemological beliefs in introductory physics, Cogn. Instruct. 12, 151 (1994).

${ }^{15}$ A. Elby and D. Hammer, On the substance of a sophisticated epistemology, Sci. Educ. 85, 554 (2001).

${ }^{16}$ D. Hammer, in Proceedings of the International School of Physics, "Enrico Fermi" Course CLVI, edited by E. F. Redish and M. Vicentini (IOS, Amsterdam, 2004), p. 301.

${ }^{17} \mathrm{M}$. Wittmann, The object coordination class applied to wavepulses: Analyzing student reasoning in wave physics, Int. J. Sci. Educ. 24, 97 (2002).

${ }^{18}$ W. Sandoval, Understanding students' practical epistemologies and their influence on learning through inquiry, Sci. Educ. 89, 634 (2005).

${ }^{19}$ R. Bromme, D. Kienhues, and E. Stahl, in Knowing, Knowledge, and Beliefs: Epistemological Studies Across Diverse Cultures, edited by M. Shine (Springer, New York, 2008), p. 423.

${ }^{20}$ S. B. Most, B. J. Scholl, E. R. Clifford, and D. J. Simons, What you see is what you set: Sustained inattentional blindness and the capture of awareness, Psychol. Rev. 112, 217 (2005).

${ }^{21}$ M. Minsky, in The Psychology of Computer Vision, edited by P. Winston (McGraw-Hill, New York, 1975), p. 211.

${ }^{22}$ D. Rumelhart and A. Ortony, in Schooling and the Acquisition of 
Knowledge, edited by R. Anderson and R. Spiro (Erlbaum, Hillsdale, NJ, 1977), p. 99.

${ }^{23}$ R. C. Schank and R. P. Abelson, Scripts, Plans Goals, and Understanding: An Inquiry into Human Knowledge Structures (Erlbaum, Hillsdale, NJ, 1977).

${ }^{24}$ G. Bateson, Steps to an Ecology of Mind: Collected Essays in Anthropology, Psychiatry, Evolution, and Epistemology (Ballantine, New York, 1972).

${ }^{25}$ E. Goffman, Frame Analysis: An Essay on the Organization of Experience (Harper and Row, New York, 1974).

${ }^{26}$ D. Tannen, in Framing in Discourse, edited by D. Tannen (Oxford University Press, New York, 1993), p. 14.

${ }^{27}$ G. MacLachlan and I. Reid, Framing and Interpretation (Melbourne University Press, Carlton, Victoria, 1994).

${ }^{28}$ E. Goffman, in The Goffman Reader, edited by C. Lemert and A. Branaman (Blackwell, Malden, MA, 1997), p. 167.

${ }^{29}$ E. Goffman, in The Goffman Reader, edited by C. Lemert and A. Branaman (Blackwell, Malden, MA, 1997), p. 149.

${ }^{30}$ D. Tannen and C. Wallat, in Framing in Discourse, edited by D. Tannen (Oxford University Press, New York, 1993), p. 57.

${ }^{31}$ M. A. van Rees, Discourse analysis and argumentation theory: The case of television talk, J. Pragmat. 39, 1454 (2007).

${ }^{32}$ K. Atkinson and T. Bench-Kapon, Artif. Intell. 171, 855 (2007).

${ }^{33}$ L. Carroll, What the tortoise said to Achilles, Mind 4, 278 (1895).

${ }^{34} \mathrm{~S}$. Toulmin, The Uses of Argument (Cambridge University Press, Cambridge, 1958).

${ }^{35}$ S. E. Newman and C. C. Marshall, Pushing Toulmin Too Far: Learning from an Argument Representation Scheme (Xerox Palo Alto Research Center, Palo Alto, CA, 1992), retrieved June 10, 2008 from http://www.csdl.tamu.edu/ marshall/toulmin.pdf

${ }^{36}$ L. Adelman, P. E. Lehner, B. A. Cheikes, and M. F. Taylor, An empirical evaluation of structured argumentation using the Toulmin argument formalism, IEEE Trans. Syst. Man Cybern., Part A. Syst. Humans 37, 340 (2007).

${ }^{37} \mathrm{U}$. Hahn and M. Oaksford, The rationality of informal argumentation: A Bayesian approach to reasoning fallacies, Psychol. Rev. 114, 704 (2007).

${ }^{38}$ J. L. Lemke, Articulating communities: Sociocultural perspectives on science education, J. Res. Sci. Teach. 38, 296 (2001).

${ }^{39}$ S. A. Southerland, E. Abrams, C. Cummins, and J. Anzelmo, Understanding students' explanations of biological phenomena:
Conceptual frameworks or p-prims?, Sci. Educ. 85, 328 (2001).

${ }^{40} \mathrm{~A}$. Watson, P. Spirou, and D. Tall, The relationship between physical embodiment and mathematical symbolism: The concept of vector (The University of Warwick, Coventry, 2003), retrieved December 2, 2009 from http://www.warwick.ac.uk/staff/David.Tall/pdfs/dot2003cwatson-spirou.pdf

${ }^{41}$ D. Tall, Introducing three worlds of mathematics, (The University of Warwick, Coventry, 2004), retrieved December 2, 2009 from http://www.warwick.ac.uk/staff/David.Tall/pdfs/dot2004a3worlds-flm.pdf

${ }^{42}$ D. Tannen, That's Not What I Meant (Ballantine, New York, 1992).

${ }^{43}$ R. Lippmann, Ph.D. thesis, University of Maryland, 2003, http:// www.physics.umd.edu/perg/dissertations/Lippmann/

${ }^{44}$ Note that our analysis presumes that students possess a group of shared framings. A mixing of different populations such as physicists and biologists or introductory students with distinctly different preparations might encounter more severe epistemological difficulties than those described here. The students we observed all appeared capable of accessing the four framings discussed in this paper.

${ }^{45} \mathrm{G}$. Lakoff and R. Nunez, Where Mathematics Comes From: How the Embodied Mind Brings Mathematics into Being (Basic Books, New York, 2001).

${ }^{46} \mathrm{~S}$. Dehaene, The Number Sense: How the Mind Creates Mathematics (Oxford University Press, New York, 1999).

${ }^{47}$ R. S. Russ, Ph.D. thesis, University of Maryland, 2006, http:// www.physics.umd.edu/perg/dissertations/Russ/

${ }^{48}$ R. S. Russ, R. E. Scherr, D. Hammer, and J. Mikeska, Recognizing mechanistic reasoning in student scientific inquiry: A framework for discourse analysis developed from philosophy of science, Sci. Educ. 92, 499 (2008).

${ }^{49}$ See Ref. 1(b), Chap. 7.

${ }^{50}$ The integrals with $x$ and $y$ omit factors that correspond to the cosine of the angle between the force and the displacement.

${ }^{51}$ T. J. Bing and E. F. Redish, Symbolic manipulators affect mathematical mindsets, Am. J. Phys. 76, 418 (2008).

52 J. Tuminaro and E. F. Redish, Elements of a cognitive model of physics problem solving: Epistemic games, Phys. Rev. ST Phys. Educ. Res. 3, 020101 (2007). 